\title{
Spontaneous resolution of nitrofurantoin-induced chronic pulmonary toxicity presenting with respiratory failure
}

The authors declare no financial disclosure

\begin{abstract}
Nitrofurantoin is one of the most common drugs implicated in drug-induced pulmonary toxicities, the manifestations of which range from dose-independent acute self-limiting reactions to chronic dose-dependent pathologies. The severity of these pulmonary adverse effects may range from trivial hypersensitivity reactions to extensive and irreversible lung fibrosis leading to respiratory failure and death. Symptomatic and supportive treatment in addition to discontinuation of the drug usually suffices for the management of mild reactions. Corticosteroids have been traditionally used to relieve the symptoms and hasten the resolution of pulmonary lesions in case of severe toxicities. However conclusive evidence in the form of controlled studies in favor of this routine use is lacking. We report a case of an elderly lady who presented to us in respiratory failure due to nitrofurantoin induced chronic lung disease. The patient's symptoms and blood gas abnormalities resolved spontaneously over the course of 6 months after stopping the drug. Radiological lesions including those suggestive of apparently permanent fibrosis also resolved during this time. Corticosteroids, inhalational or systemic, were not used. Spontaneous normalization of clinico-radiological features in nitrofurantoin induced chronic lung disease presenting with respiratory failure has rarely been reported previously. The related literature is also reviewed.
\end{abstract}

Key words: nitrofurantoin, pulmonary, lung, drug-induced ILD, respiratory failure, corticosteroids

Adv. Respir. Med. 2017; 85: 333-338

\section{Introduction}

Nitrofurantoin is one of the most common antimicrobials used for prophylaxis as well as treatment of urinary tract infections (UTIs) [1]. The Infectious Diseases Society of America and the European Society for Microbiology and Infectious Diseases recommend nitrofurantoin as one of the first line antibiotics in uncomplicated UTIs, given the low cost of therapy, efficacy as well as its positive impact on antimicrobial resistance patterns [1]. However, nitrofurantoin is also one of the most common drugs to be reported for a variety of pulmonary, hepatic and peripheral nervous system adverse effects [2-4]. The spectrum of pulmonary toxicities ranges from acute hypersensitivity reactions to chronic dose-dependent pathologies like chronic interstitial pneumoni- tis, fibrosis and organizing pneumonia [2-4]. In view of these possible side effects and available alternative medicines, the American Geriatrics Society in a 2015 update listed nitrofurantoin as a "potentially inappropriate medication" for prolonged use in adults 65 years of age and older [4].

Mild pulmonary reactions due to nitrofurantoin usually resolve spontaneously after the drug is discontinued [2, 3]. The literature on the management of nitrofurantoin-induced severe chronic pulmonary toxicities is meager. Corticosteroids, in addition to supportive therapy, are often used in these patients, even though the evidence in support of such routine application in the form of controlled trials is lacking [2, 3]. We report a case of an elderly lady who presented to us with respiratory failure as a result of severe pulmonary toxicity due to prolonged nitrofurantoin use.

Address for correspondence: Elias Mir, SKIMS Medical College, Bemina, 190018, Srinagar, India, e-mail: eliasjnmc@gmail.com

DOI: 10.5603/ARM.2017.0057

Received: 04.09.2017

Copyright (C) 2017 PTChP

ISSN 2451-4934 


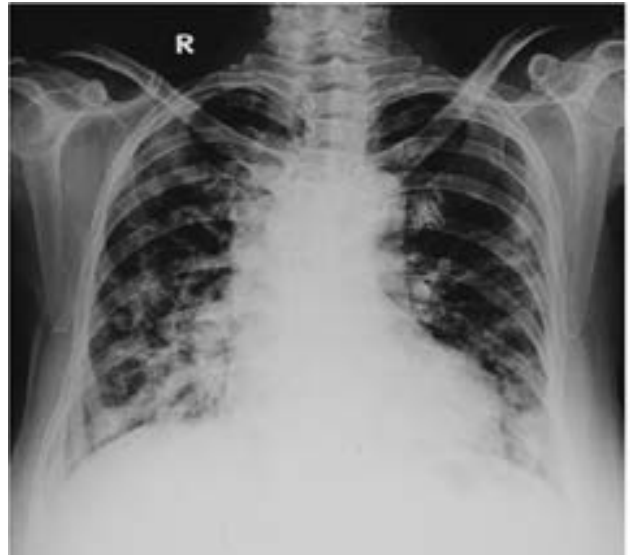

A

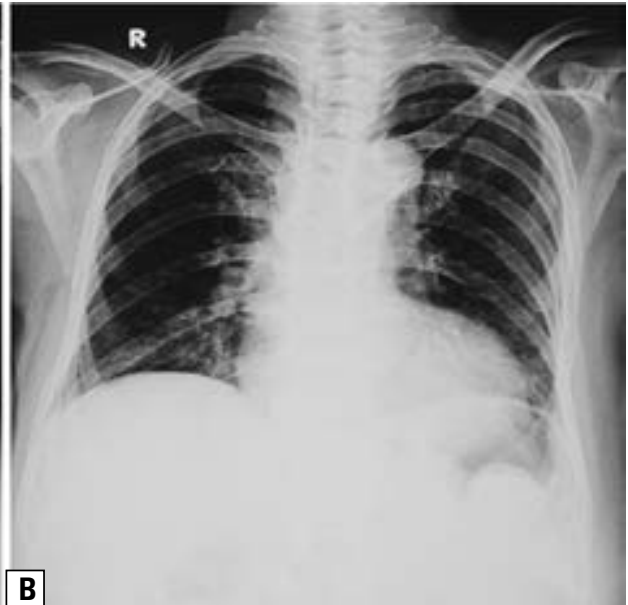

B

Figure 1. A - chest radiograph at presentation showing consolidations and reticulonodular pattern in bilateral mid and lower zones; B — chest radiograph after 6 months of presentation showing near-normalization of radiological abnormalities

After the drug was stopped, the patient improved gradually without the use of inhaled or systemic corticosteroids.

\section{Case report}

An 85-year-old never-smoker, HIV-negative lady was referred to our hospital for evaluation of her progressive respiratory symptoms including non-productive cough and breathlessness on exertion, for the preceding one month. The patient did not report fever, chest pain or wheeze. There was no history of exposure to fumes or contact with domestic pets. Her past history was unremarkable except for recurrent urinary tract infections for which she was taking regular nitrofurantoin prophylaxis (50 mg once daily) for the preceding three years.

On clinical examination the patient was in respiratory distress. She had tachycardia (pulse rate of 104/minute) and tachypnea (respiratory rate of $28 /$ minute). The woman was hypoxemic with an $\mathrm{SpO}_{2}$ of $78 \%$ while breathing room air. Chest examination revealed symmetrical diaphragmatic excursion with bilateral fine rales on inspiration. The rest of the systemic examination was unremarkable.

Blood counts, hemoglobin, kidney/liver function tests and routine urine analysis did not reveal any abnormality. Arterial blood gas (ABG) analysis confirmed hypoxemia with $\mathrm{PaO}_{2}$ of $51 \mathrm{~mm}$ $\mathrm{Hg}$ while breathing room air. Chest radiograph at the time of presentation (Fig. 1A) demonstrated bilateral peripheral reticulonodular opacities and consolidations in mid and lower zones with relative sparing of upper zones. High resolution computed tomography (HRCT) of the thorax (Fig. 2) revealed bilateral, patchy, sub-pleural and peribronchial consolidations, reticular opacities and areas of mosaic lung attenuation along with traction bronchiectasis and architectural distortion. Multiple sputum samples and a bronchoalveolar lavage (BAL) were negative on gram stain, Ziehl-Neelsen stain for acid fast bacilli and $\mathrm{KOH}$ mounts on microscopy. Sputum and BAL cultures for aerobic bacteria, fungi and Mycobacterium tuberculosis were also negative. Pulmonary function test (PFT) revealed a restrictive pattern with reduced forced vital capacity (FVC) (55\% of the predicted value) and normal FEV1/FVC (79\%). Diffusion capacity for carbon monoxide (DLCO) was also reduced (59\% of the predicted value). Serology for antinuclear, anti-double stranded DNA, anti Scl 70, anti-citrullinated cyclic polypeptide and antineutrophil cytoplasmic antibodies (both perinuclear and cytoplasmic) was negative.

In view of a history of exposure to a drug known to cause pulmonary toxicity, temporal association of drug exposure with the clinical condition, typical clinico-radiological presentation and a negative workup for infectious and connective tissue diseases, nitrofurantoin was presumed to be the causative agent for the disease. The patient's score on the Naranjo Adverse Drug Reaction Probability Scale [5] was 7, which placed the patient in a "probable adverse drug reaction" category.

Nitrofurantoin was immediately stopped at admission. During her stay at the hospital, the patient was given only symptomatic treatment with inhaled salbutamol along with supplemental oxygen. Neither corticosteroids, inhalational or systemic, nor antibiotics were used. The clinical condition of the patient gradually im- 


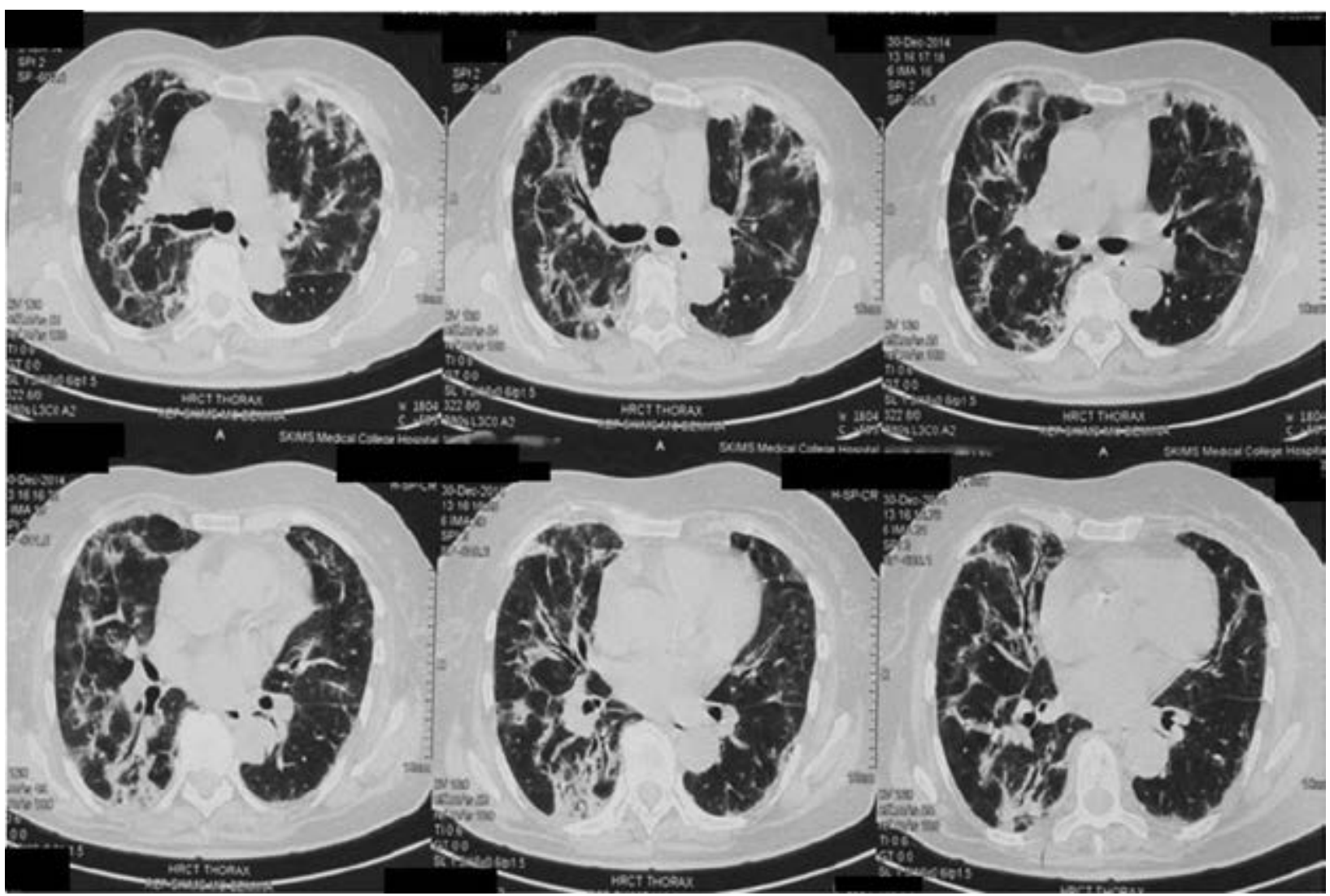

Figure 2. HRCT at presentation showing bilateral sub-pleural reticular opacities, patches of subpleural and peribronchial consolidation, mosaic lung attenuation and architectural distortion

proved during her stay at the hospital. She was discharged with continuous oxygen therapy at home and advised regarding strict avoidance of nitrofurantoin and regular outpatient follow up. The patient showed further gradual improvement at home. Over a course of six months, she became asymptomatic. Her respiratory failure also resolved, with oxygen saturation and $\mathrm{PaO}_{2}$ improving to $97 \%$ and $77 \mathrm{~mm} \mathrm{Hg}$, respectively, while breathing room air. Lung function tests including spirometry and diffusion capacity were also normal for her age, sex and height at the end of 6 months. Chest radiograph (Fig. 1B) and HRCT of the thorax (Fig. 3) repeated after 6 months revealed nearly complete resolution of radiological abnormalities.

\section{Discussion}

Nitrofurantoin-induced lung disease (NILD) was first reported by Fisk in 1957 who described an acute self-limiting reaction to nitrofurantoin characterized by fever, dyspnea, cough, chest pain, rash, arthralgia, tachycardia and tachypnea [6]. Chronic NILD is less common than the acute form, and can appear months to even years after initiation of treatment [2, 3]. It most commonly occurs in older women due to the higher prevalence of UTIs in this age group. The usual presentation is gradual onset of cough, progressive dyspnea, myalgia, weight loss and fatigue. Clinical findings include bilateral inspiratory fine rales and clubbing. Chest radiograph commonly reveals bilateral interstitial infiltrates predominantly in the lower zones. Pulmonary function test (PFT) may reveal a restrictive pattern with reduced diffusion capacity. High resolution computed tomography (HRCT) scan findings include ground-glass attenuation, inter- and intra-lobular septal thickening, traction bronchiectasis, honeycombing and organizing pneumonia [2, 3]. Our patient therefore presented with a chronic NILD with classical clinico-radiological features. Lung biopsy was not done. However, radiological picture was suggestive of interstitial inflammation (reticular opacities), fibrosis (architechtural distortion and traction bronchiectasis), organizing pneumonia (peripheral, subpleural and peribronchial consolidations) as well as a probable small airway involvement (mosaic attenuation).

We searched PubMed for cases of nitrofurantoin-induced lung adverse lung reactions leading 


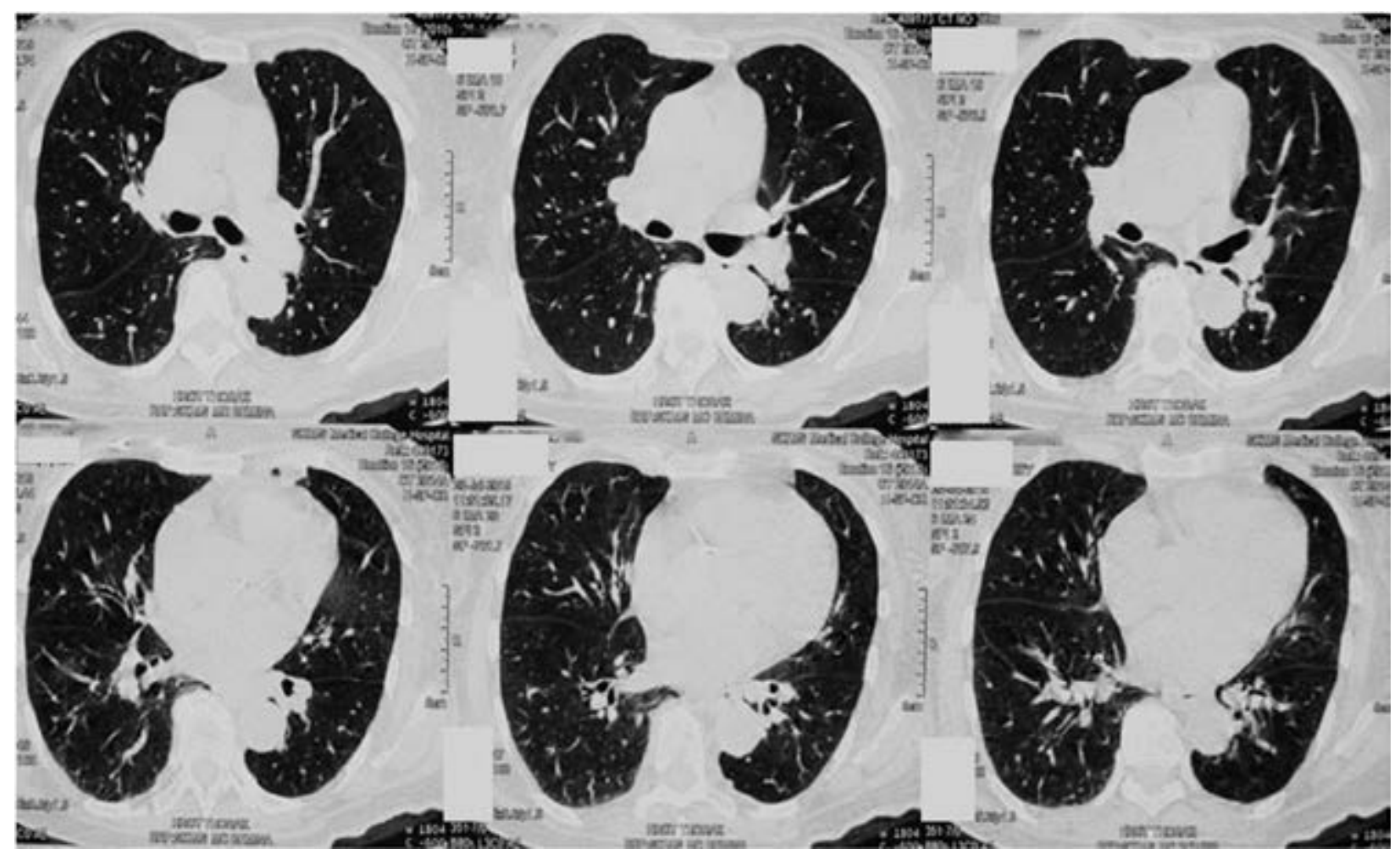

Figure 3. HRCT of thorax after 6 months of presentation showing near-complete resolution of radiological abnormalities

to respiratory failure that resolved spontaneously using the key words "NITROFURANTOIN", "LUNG", "PULMONARY", "CORTOCOSTEROIDS" and "ILD". We could find only 4 such cases [7-10] published in English since 1953 (Table 1). All of these patients were females and almost all of them had a complete spontaneous normalization of clinico-radiological picture. The time of resolution varied between 3 to 4 months in 3 patients while 1 patient had nitrofurantoin-induced respiratory arrest only without parenchymal involvement, which resolved in a day without the use of corticosteroids [7-10]. Histopathology revealed granulomatous pneumonitis in the only patient who underwent a lung biopsy [9]. Our patient showed complete resolution of symptoms, near-normalization of blood gas parameters as well as radiological changes in 6 months. Surprisingly, radiological features suggestive of irreversible fibrosis like traction bronchiectasis and architectural distortion also resolved with time. Such resolution of apparently permanent fibrosis has been previously reported [12].

NILD-related mortality is well documented [2, 3, 13]. In our patient careful history and prompt drug withdrawal avoided further morbidity as well as an unnecessary lung biopsy. This case highlights the need for increased sensitivity towards nitrofurantoin-induced lung adverse events especially in older adults after prolonged use. The patients may require continued surveillance for these toxicities in the form of periodic radiographs, pulmonary function tests and arterial blood gas monitoring.

The role of corticosteroids in severe NILD is controversial. It is impossible to conduct controlled studies in these patients to substantiate the evidence for or against the routine use of steroids. Many NILD patients have succumbed in spite of corticosteroid therapy [2, 3, 13]. Even in patients already on immunosuppressive therapy, fresh nitrofurantoin-related pulmonary adverse events including fatal pulmonary hemorrhages have been reported [13]. Regular follow up avoided many corticosteroid-related side effects in our patient.

We can conclude through this case and other case reports [7-10] that there is at least a subset of patients with advanced chronic NILD who will show spontaneous resolution of clinico-radiological abnormalities after drug withdrawal without corticosteroid use. We recommend strict follow up with monitoring of lung functions and blood gases after drug withdrawal until complete normalization in such patients. 


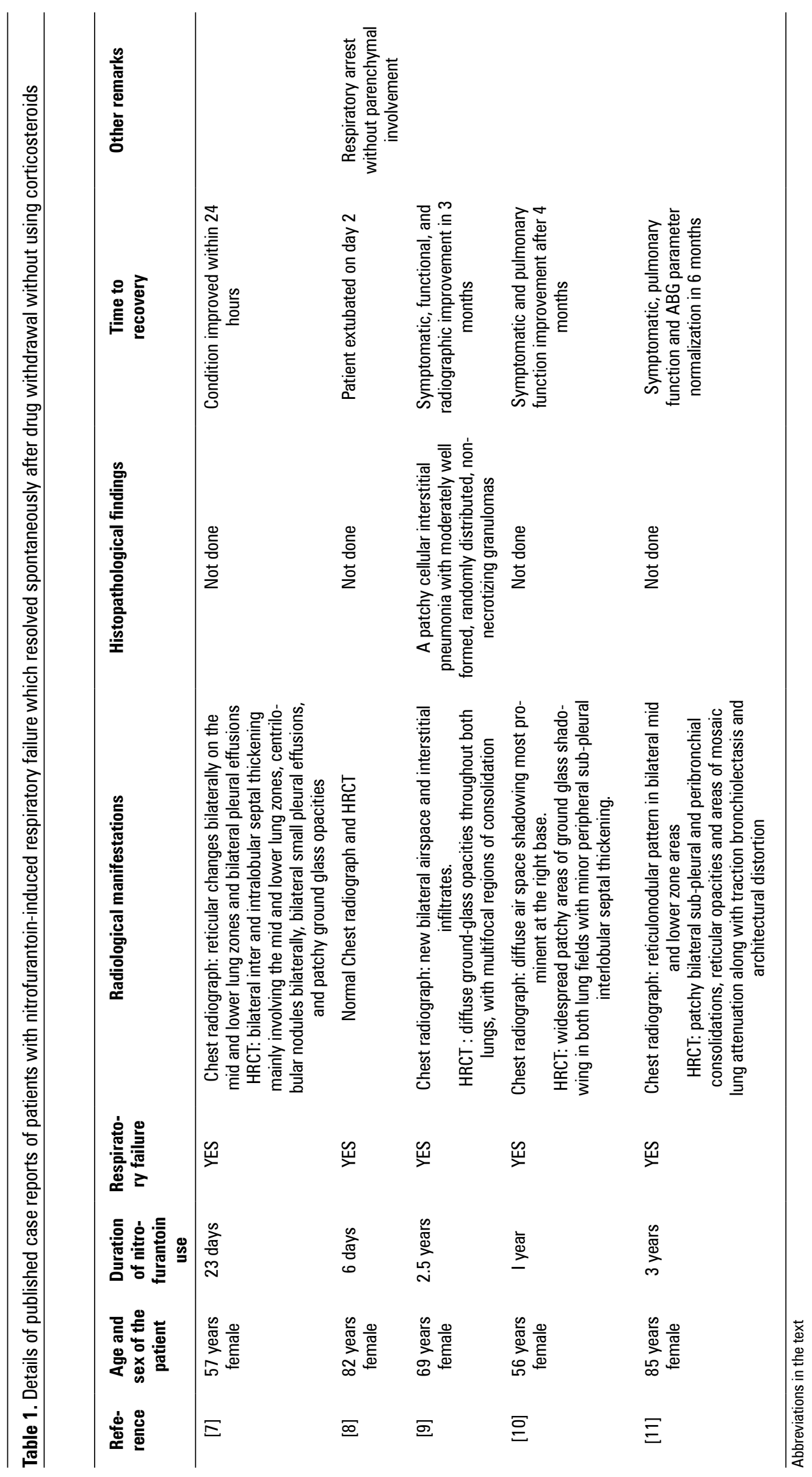




\section{Conflict of interest}

\section{The authors declare no conflict of interest.}

\section{References:}

1. Gupta K, Hooton TM, Naber KG, et al. Infectious Diseases Society of America, European Society for Microbiology and Infectious Diseases. International clinical practice guidelines for the treatment of acute uncomplicated cystitis and pyelonephritis in women: A 2010 update by the Infectious Diseases Society of America and the European Society for Microbiology and Infectious Diseases. Clin Infect Dis. 2011; 52(5): e103-e120, doi: 10.1093/cid/ciq257, indexed in Pubmed: 21292654.

2. Holmberg L, Boman G, Böttiger LE, et al. Adverse reactions to nitrofurantoin. Analysis of 921 reports. Am J Med. 1980; 69(5): 733-738, indexed in Pubmed: 7435512.

3. Sovijärvi AR, Lemola M, Stenius B, et al. Nitrofurantoin-induced acute, subacute and chronic pulmonary reactions. Scand J Respir Dis. 1977; 58(1): 41-50, indexed in Pubmed: 841294.

4. By the American Geriatrics Society 2015 Beers Criteria Update Expert Panel. American Geriatrics Society 2015 Updated Beers Criteria for Potentially Inappropriate Medication Use in Older Adults. J Am Geriatr Soc. 2015; 63(11): 2227-2246, doi: 10.1111/jgs.13702, indexed in Pubmed: 26446832.

5. Busto U, Naranjo CA, Sellers EM, et al. A method for estimating the probability of adverse drug reactions. Clin Pharmacol Ther. 1981; 30(2): 239-245, indexed in Pubmed: 7249508.
6. FISK AA. Brief recording: anaphylactoid reaction to nitrofurantoin. N Engl J Med. 1957; 256(22): 1054, doi: 10.1056/ NEJM195705302562207, indexed in Pubmed: 13451992.

7. Livanios K, Karampi ES, Sotiriou A, et al. Nitrofurantoin-induced acute pulmonary toxicity. Respirol Case Rep. 2016; 4(1): 25-27, doi: 10.1002/rcr2.131, indexed in Pubmed: 26839698.

8. Çoban H. Respiratory arrest due to nitrofurantoin treatment in the absence of pulmonary pathology. J Basic Clin Pharm. 2015; 6(4): 115-116, doi: 10.4103/0976-0105.168050, indexed in Pubmed: 26692737.

9. Sakata KK, Larsen BT, Boland JM, et al. Nitrofurantoin-Induced Granulomatous Interstitial Pneumonia. Int J Surg Pathol. 2014; 22(4): 352-357, doi: 10.1177/1066896913492849, indexed in Pubmed: 23812013.

10. Weir M, Daly GJ. Lung toxicity and Nitrofurantoin: the tip of the iceberg? QJM. 2013; 106(3): 271-272, doi: 10.1093/qjmed/ hcs129, indexed in Pubmed: 22836192.

11. Mir E, Malik JA, Lone SA, et al. Spontaneous resolution of nitrofurantoin-induced chronic pulmonary toxicity presenting with respiratory failure. Adv Respir Med. 2017; 85: 333-338. doi: 10.5603/ARM.2017.0057, indexed in Pubmed: 29288483.

12. Sheehan RE, Wells AU, Milne DG, et al. Nitrofurantoin-induced lung disease: two cases demonstrating resolution of apparently irreversible CT abnormalities. J Comput Assist Tomogr. 2000; 24(2): 259-261, indexed in Pubmed: 10752888.

13. Vincent F, Bensousan TA. Re: Nitrofurantoin-induced pulmonary hemorrhage in a renal transplant recipient receiving immunosuppressive therapy: case report and review of the literature. J Urol. 1995; 154(1): 214, indexed in Pubmed: 7776431. 